\title{
Fertility preservation in $>1,000$ patients: patient's characteristics, spectrum, efficacy and risks of applied preservation techniques
}

\author{
Barbara Lawrenz $\cdot$ Julia Jauckus $\cdot$ Markus S. Kupka • \\ Thomas Strowitzki $\cdot$ Michael von Wolff
}

Received: 31 August 2010/Accepted: 9 November 2010/Published online: 1 December 2010

(C) Springer-Verlag 2010

\begin{abstract}
Introduction Data on the characteristics of female patients counselled for fertility preservation and the efficacy and risk of the applied procedures are still poor. We therefore analysed the registry of a network of 70 infertility centers which are involved in fertility preservation in Germany, Switzerland and Austria, called FertiPROTEKT (hhtp://www.fertiprotekt.eu).

Materials and methods 1,280 counselled patients (15-40 years) were analysed regarding characteristics and different fertility preservation treatments before cytotoxic therapy in 2007-2009.

Results $34.8 \%$ of the counselled patients were diagnosed with breast cancer, $30.5 \%$ with Hodgkin's lymphoma, $25.4 \%$ with other malignancies and $9.3 \%$ with non-malignant diseases. $89.6 \%$ of the treated breast cancer patients were 25-40 years of age, and $87.5 \%$ of the lymphoma patients were 15-30 years of age. At the time of counselling, 85.3\%
\end{abstract}

B. Lawrenz

University Women's Hospital, Tübingen, Germany

J. Jauckus · T. Strowitzki

Department of Gynaecological Endocrinology and Reproductive

Medicine, University Women's Hospital, Heidelberg, Germany

M. S. Kupka

University Women's Hospital, Klinikum Maistrasse,

Munich, Germany

M. von Wolff $(\bowtie)$

Department of Gynaecological Endocrinology and Reproductive

Medicine, University Women's Hospital, Effingerstrasse 102,

3010 Bern, Switzerland

e-mail: Michael.vonWolff@insel.ch of the breast cancer patients and $92.7 \%$ of the lymphoma patients were childless.

1,080 patients received a single or combined therapy such as GnRH agonists $(n=823)$, cryopreservation of ovarian tissue $(n=500)$, ovarian stimulation $(n=221)$ and transposition of the ovaries $(n=24)$. Only one severe complication, requiring postponement of the chemotherapy, was documented. In stimulated patients, 2,417 oocytes (mean $n=11.6, \mathrm{SD} \pm 7.7$ ) were received. Fertilisation rate per received oocyte was $61.3 \%$.

Conclusions Fertility preservation programmes mainly involve women without children, diagnosed with breast cancer or Hodgkin's lymphoma. Fertility preservation techniques can be applied with low risk. The limited and age-dependant success rate of the different therapies require individualised approaches of single or combined fertility preservation techniques.

Keywords Fertility preservation - IVF - Cancer - Oocyte GnRH $\cdot$ Cryopreservation

\section{Introduction}

Increasing survival rates after cancer, new reproductive techniques and growing interest regarding the quality of life after cancer and after chemotherapy to treat other benign systemic disease have brought the possibilities of fertility preserving treatment to the centre of attention of oncologists and the affected female patients. Many of these women suffer from infertility and premature menopause as a result of chemotherapy and/or radiotherapy to the small pelvis.

As approximately $70 \%$ of patients indicate a prospective desire for children after completion of chemotherapy [1,2], 
fertility preservation procedures are of particular relevance, however, only a few oncologists refer patients to a specialist in reproductive medicine to take steps to preserve fertility [3].

Methods of fertility preservation by ovarian protection include the administration of gonadotrophin agonists (GnRH-a) [4, 5], cryoconservation with later re-transplantation of the ovarian tissue [6] and hormonal stimulation to retrieve oocytes with subsequent cryoconservation of fertilised or unfertilised egg cells and transposition of the ovaries before radiotherapy to the small pelvis [7].

The various methods can also be combined with one another [8]. Experience with these techniques and data about their efficacy and risks are, however, still limited, which may be an important reason for the limited growth of fertility preservation procedures. The limited data hinders balanced patient counselling and the individual integration of fertility preservation techniques into the oncological treatment regimen.

Extensive representative registers from networks on the implementation of fertility protection techniques allow the possibility of reducing this data deficit. The FertiPROTEKT network (http://www.fertiprotekt.eu) [9] is one particularly extensive network with a register. The network was established in 2006 and includes around 70 centres in Germany, Switzerland and Austria. The aim is the pooling of expertise from oncologists, rheumatologists and reproductive medicine specialists, and the implementation of a comprehensive national care structure and development of obligatory treatment recommendations and standardised counselling structures. Compulsory documentation of all patients and treatments has been made since 2007 .

We analysed the data from this register for the first time to obtain solid data on the characteristics of the counselled and treated patients, about the efficacy of ovarian stimulation treatment in particular, and about the complication rates from performing fertility preservation techniques.

\section{Materials and methods}

The retrospective analysis includes FertiPROTEKT network data from patients aged between 15 and 40 years of age, who presented for counselling on and treatment with fertility preservation techniques prior to receiving chemotherapy between 01.01.2007 and 31.12 2009 in member centres of the network.

The 1,388 registers were screened and checked. Missing details, which should have been analysed in this study or inconsistent data were not considered. $9.2 \%$ of the records ( $n=108$ ) were excluded, and 1,280 records remained for the analysis.
Specialists in reproductive medicine from the network advised the patients and their partners depending on tumour characteristics, ovarian toxicity of the chemotherapy, the patient's wishes, and the counselling and mandatory treatment standards prepared by the network with regard to risks, chances of pregnancy and cost of the fertility preserving techniques. The fertility preservation techniques were incorporated into the treatment regimen in agreement with the responsible doctors.

The treatment recommendations are available on the network's website (http://www.fertiprotekt.eu), and can be summarised as follows.

GnRH analogues (GnRH-a) used are goserelin (Zoladex; Astra-Zeneca, Wedel, Germany) or leuprorelin (Enantone; Takeda Pharma, Aachen, Germany), as a depot injection for the duration of the chemotherapy.

Removal of ovarian tissue, when possible, is performed laparoscopically and coagulation of the removed tissue is avoided. The amount of tissue removed depends on the anticipated damage to the ovaries. Ovarian tissue was cryopreserved by slow freezing.

Either an agonist-short-protocol (GnRH-a), an antagonist protocol (GnRH-anta) or in the case of stimulation in the luteal phase, a modified antagonist protocol (mGnRHanta) [10] are used for hormonal stimulation to retrieve oocytes. Vaginal follicle puncture is performed $36 \mathrm{~h}$ after induction of ovulation. If the patient has a long-term partner, the oocytes are fertilised and cryoconserved at the pronuclear stage (PN) according to German national law.

In the case of ovarian transposition, the ovaries are mobilised uni-or bilaterally in order to relocate them cranially and laterally and to fix them to the peritoneum.

\section{Results}

Patients

The indication for counselling was a malignancy in $90.7 \%$ of the patients and a benign systemic illness in $9.3 \%$. The mean age of the counselled and treated patients was 27.8 and 27.4 years, respectively (range 15-40, SD \pm 7.0 and range $15-40, \mathrm{SD} \pm 6.8$ ).

The two most common malignancies were breast cancer and lymphoma, and therefore the data was analysed separately for these disorders. $89.6 \%$ of the breast cancer patients who decided on fertility preservation treatment were between 25 and 40 years of age, and $87.5 \%$ of the lymphoma patients were between 15 and 30 years of age. At the time of counselling, $85.3 \%$ of the breast cancer patients and $92.7 \%$ of the lymphoma patients were childless. 
The characteristics (disease, parity, age) of the counselled and treated patients are shown in Table 1.

Implementation of fertility preservation techniques

After counselling, a total of $84.3 \%$ of all patients decided on one or on a combination of various fertility preservation methods. This equated to $77.6 \%$ of breast cancer and $92.3 \%$ of lymphoma patients.

A total of 1,568 procedures, alone or in combination, were performed.

The most common treatment was administration of GnRH-a ( $n=823)$, followed by removal of ovarian tissue for cryoconservation $(n=500)$. Ovarian stimulation to retrieve oocytes was the third most common method ( $n=221$ ). Figure 1 shows the utilization of the methods, also differentiated for patients with all indications and for patients with breast cancer or lymphoma.

In the patient group who were only treated with one fertility preservation technique, administration of $\mathrm{GnRH}-\mathrm{a}$ ( $n=471)$ was-in analogy to all the treatments performed - the most commonly used technique, followed by cryoconservation of ovarian tissue $(n=241)$ and ovarian stimulation $(n=72)$. The use of the techniques varied depending on the different tumour characteristics.

To increase the efficacy of the fertility preservation techniques, they were combined with one another to some

Table 1 Characteristics (disease, parity, age) of the counselled and treated patients

\begin{tabular}{lrr}
\hline & \multicolumn{2}{l}{ Number of patients } \\
\cline { 2 - 3 } & Counselling & \multicolumn{1}{c}{ Treatment } \\
\hline Counselling indication & $446(34.8 \%)$ & $346(32.0 \%)$ \\
Breast cancer & $390(30.5 \%)$ & $360(33.3 \%)$ \\
Lymphoma & $325(25.4 \%)$ & $269(24.9 \%)$ \\
Other malignant disease ${ }^{\mathrm{a}}$ & $119(9.3 \%)$ & $105(9.7 \%)$ \\
Benign illness ${ }^{\mathrm{b}}$ & $1,280(100 \%)$ & $1,080(100 \%)$ \\
Total & & \\
Number of children & $1,102(86.1 \%)$ & $944(87.4 \%)$ \\
No children & $178(13.9 \%)$ & $136(12.6 \%)$ \\
1 or more children & $1,280(100 \%)$ & $1,080(100 \%)$ \\
Total & & \\
Age (years) & $468(36.5 \%)$ & $423(39.1 \%)$ \\
$15-25$ & $655(51.2 \%)$ & $534(49.4 \%)$ \\
$26-35$ & $157(12.3 \%)$ & $123(11.4 \%)$ \\
$36-40$ & $1,280(100 \%)$ & $1,080(100 \%)$ \\
Total &
\end{tabular}

${ }^{a}$ Cancer of the gastrointestinal tract, gynaecological cancers, germcell carcinomas, melanomas, neurological cancers, sarcoma

b Benign haematological disorders, Turner syndrome, immunological disorders, auto-immune disorders, systemic lupus erythematosus extent. The most commonly used combination $(n=211)$ was administration of a GnRH-a with removal of ovarian tissue, followed by administration of GnRH with hormonal stimulation ( $n=116$ ). Other combinations were: GnRH-a plus ovarian stimulation plus removal of ovarian tissue, GnRH-a plus ovarian transposition plus ovarian stimulation and ovarian stimulation plus removal of ovarian tissue.

Hormonal stimulation to remove oocytes

Data from 205 follicle punctures were analysed.

The mean stimulation duration was 10.9 days (SD \pm 2.6); the mean stimulation dose per day was $225 \mathrm{IE} /$ day $(\mathrm{SD} \pm 59 \mathrm{IE} /$ day) with a mean total stimulation medication dose of 2,465 IE (SD $\pm 875 \mathrm{IE})$.

A total of 2,417 oocytes were removed (range 0-41, mean $n=11.6$, median $n=10$, STD $\pm 7.7,25 \%$ quartile $n=6,75 \%$ quartile $n=15$ ), 932 oocytes could be fertilised and 773 were cryoconserved unfertilised.

ICSI was performed with all oocytes from 125 follicle punctures, and the fertilised oocytes were cryoconserved in the pronuclear stage (Fig. 2). 1,375 oocytes were retrieved (range $1-41$, mean $n=11.0$, median $n=10.0, \mathrm{SD} \pm 6.6$, $25 \%$ quartile $n=6,75 \%$ quartile $n=14$ ) and 843 were successfully fertilised (range $1-27$, mean $n=6.7$, median $n=6.0, \mathrm{SD} \pm 4.8,25 \%$ quartile $n=3,75 \%$ quartile $n=9)$. The fertility rate per removed oocyte was $61.3 \%$.

\section{Complications}

Complications of fertility preservation techniques were:

- Events which led to the postponement of the start of chemotherapy

- Termination of ovarian stimulation

- Ovarian stimulation without oocyte retrieval or without fertilisation.

There were 8 events from a total of 1,568 fertility preservation procedures which were classified as complications, which is equivalent to a risk of $0.74 \%$ per treated patient. The risk of a complication occurring was $2.9 \%$ per stimulated patient during the course of ovarian stimulation, $0.4 \%$ per patient for ovarian tissue removal and $0.08 \%$ per treated patient when the start of chemotherapy was postponed.

The complications and their consequences for the start of chemotherapy are listed in Table 2.

\section{Discussion}

Depending on the age of the patient and the agents used, chemotherapy can lead to damage to ovarian function and 
Fig. 1 Fertility preservation techniques, for all indications and for breast cancer and lymphoma. GnRHa administration of a $\mathrm{GnRH}$ analogues, KryoOvar removal of ovarian tissue for cryoconservation, Ov.Stim ovarian stimulation to remove oocytes, Transp.Ovar transposition of the ovaries

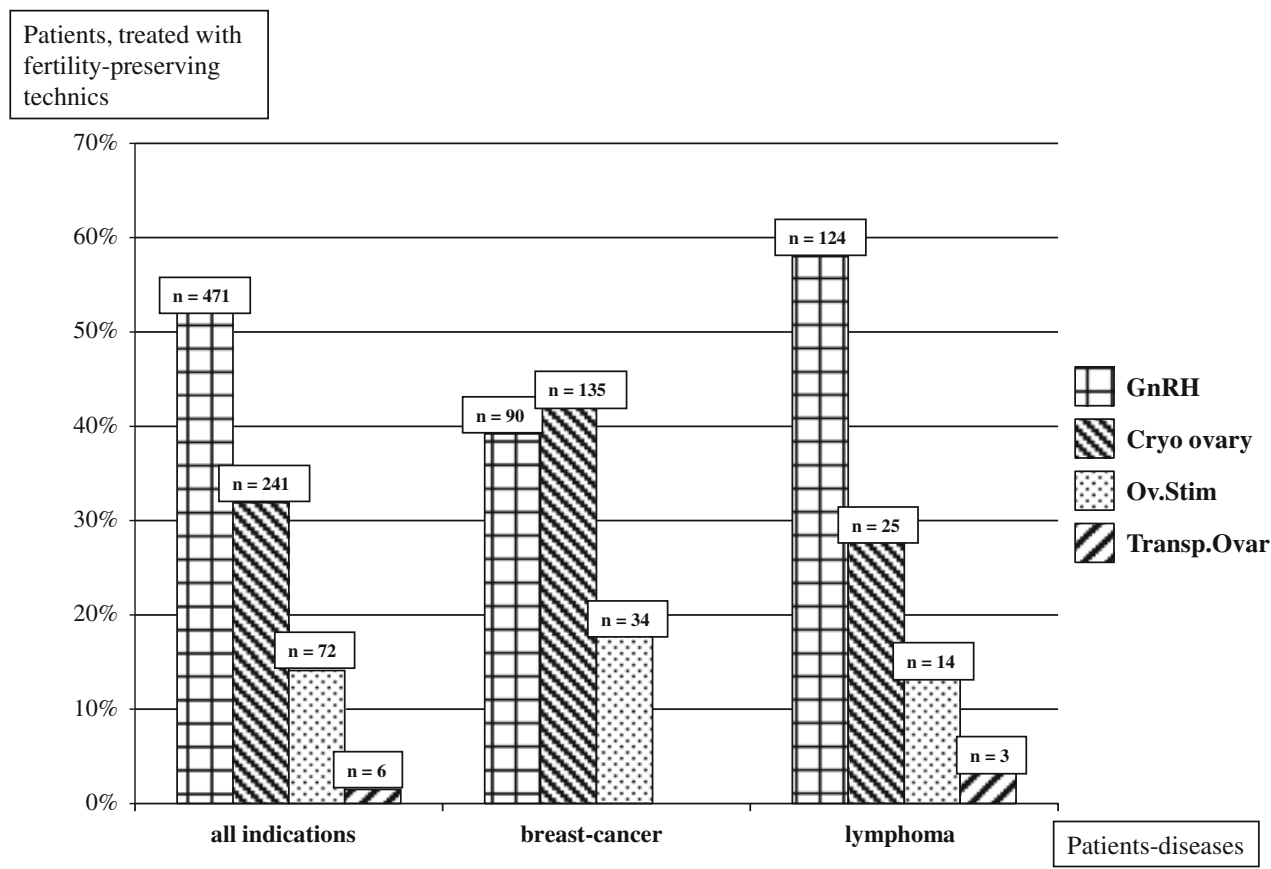

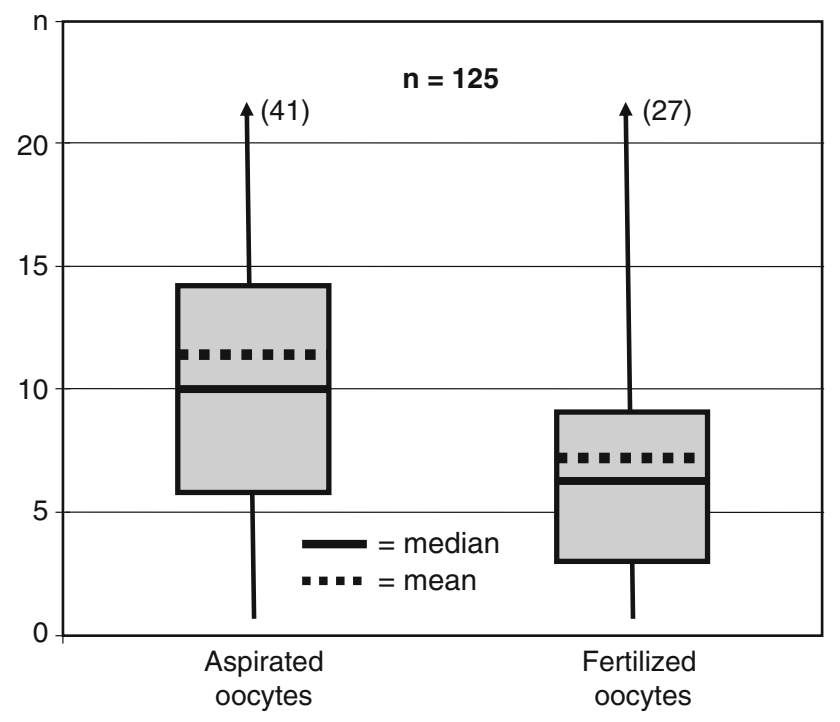

Fig. 2 Removed oocytes and successfully fertilised oocytes (pronuclear stage) by ICSI after ovarian stimulation per patient

loss of fertility [11-13]. The implementation of fertility preservation techniques in the treatment regimen is therefore of great importance. Analysis of the data from the FertiPROTEKT network revealed for the first time both the counselling indications, age distribution and parity of the counselled and treated patients and also the choice, efficacy and safety of the fertility preservation methods prior to chemotherapy in a large number of patients. These data can be useful when developing fertility protection programmes, for example to better define the patient structure and target groups, and also to help when counselling couples. The data also reflect the realistic situation in a large multicentre programme. As highly specialised as well as less specialised centres are represented in this programme, the success and complication rates described depict realistically achievable targets.

Of the patients who presented for counselling in the FertiPROTEKT network and who underwent fertility protection treatments, $86.1 \%$ (counselling) and $87.4 \%$ (treatment) were childless, and at counselling and treatment had a mean age of 27.83 and 27.35 years, respectively. A study by Klock et al. [14] also showed a prevalence of childless patients at counselling and treatment.

We recorded the age distribution of the counselled and fertility protection-treated patients independently of the choice of fertility preservation technique. This realistically reflects the age distribution of the patients for whom a need for fertility preservation counselling exists. Thus, patients who decided on cryoconservation of an entire ovary were, on average, 25.4 years of age (range 9-37) [15]; in contrast, patients who underwent hormonal stimulation to retrieve oocytes were $32.3 \pm 5.0$ years of age [16]. The mean age of the our collective of treated women was 27.35 years, yet $89.6 \%$ of the women with breast cancer were aged $25-40$ years and $87.5 \%$ of women with a lymphoma were aged 15-30.

The largest group of counselled patients was made up of patients with breast cancer (34.8\%), followed by patients with a lymphoma (30.5\%). In contrast, the most treatments within one disease group were performed on lymphoma 
Table 2 Complications during fertility preservation treatments

\begin{tabular}{llll}
\hline Age (years) & Illness & Complication & Postponement of chemotherapy \\
\hline $\begin{array}{l}\text { Complications during ovarian stimulation } \\
36\end{array}$ & & No \\
19 & AML & Termination of stimulation & No \\
34 & Breast cancer & Termination of stimulation & No \\
37 & Medulloblastoma & No follicle puncture followed & No \\
36 & SLE & No egg cells retrieved & No \\
27 & Hodgkin's lymphoma & No egg cells retrieved & No \\
Complications during & Novarian tissue removal for cryoconservation & No \\
19 & Nephroblastoma & No tissue removal possible due to adhesions & Postponement of start \\
34 & Lymphoma & Laparotomy because of secondary haemorrhage & of chemotherapy by 5 days \\
\hline
\end{tabular}

$A M L$ acute myeloid leukaemia, SLE systemic lupus erythematosus

patients $(33.3 \%)$. This data is in agreement with other studies [14-19].

Up to now, the choice of fertility preservation technique has only been studied in small collectives. Oktay et al. [18] and Klock et al. [14] evaluated data from 462 and 65 patients, respectively: in patients who decided on fertility preservation treatment, 50.6 and $85 \%$ underwent embryo cryoconservation, respectively, and 26.6 and $4.9 \%$ underwent removal of ovarian tissue, respectively. Our data analysis shows that $26.6 \%$ of breast cancer patients and $16.1 \%$ of lymphoma patients decided on hormonal stimulation to retrieve oocytes, and $60.4 \%$ of breast cancer patients and $33.3 \%$ of lymphoma patients for ovarian tissue removal. The reason for the high proportion of patients choosing cryopreservation of ovarian tissue instead of ovarian stimulation might be the fear of breast cancer patients of a negative influence of the hormonal stimulation upon the prognosis of the breast cancer, the higher cost of ovarian stimulation and the time consuming ovarian stimulations. However, as shown in this paper, ovarian stimulation and cryopreservation of fertilised oocytes is a well established and successful technique and should be the first choice in fertility preservation programmes.

Neither of the studies referred to above $[14,18]$ recorded administration of GnRH-a. Up to now, the efficacy of GnRH-a application for ovarian protection is still controversial. A recently published review by Dittrich et al. [23] summarises the inconsistent data. Despite the unclear efficacy, this was the most common treatment option in our collective, as an individual treatment as well as in combination, followed by ovarian tissue removal and hormonal stimulation. Removal and transplantation of ovarian tissue has also been performed frequently, as the procedure is performed within a few days and as it has already been shown to be successful, though still experimental [24].
No other investigation includes the combination of fertility preservation techniques. The combination of an invasive method (ovarian tissue removal, hormonal stimulation) with GnRH-a does not increase the intervention risk for the patient. Huober-Zeeb et al. [8] showed that the combination of ovarian tissue removal with subsequent hormonal stimulation can be carried out with good efficacy and without threat to the patient.

Implementation without additional risks for the patient is of great importance for the integration of fertility preservation techniques into the oncological treatment concept; therefore, recording of complications is intrinsically important.

Of the 500 patients who underwent ovarian tissue removal, perioperative complications occurred in only $0.4 \%$. This low complication rate is confirmed in work by Rosendahl et al. (4.3\%) [15] and Oktay et al. (0\%) [20].

Ovarian stimulation to remove oocytes can also be performed with a very low risk for the patient: none of our collective developed ovarian hyperstimulation syndrome (OHSS) which led to postponement of the start of chemotherapy. In $2.9 \%$ of stimulated patients, stimulation had to be stopped because of low response, or because no egg cells could be removed during follicle puncture. Other studies with small case numbers [14, 16-18] report termination or low response rates as being between 7.1 and $18 \%$.

Our results from ovarian stimulation treatment before chemotherapy are both comparable with the results from other studies, which to some extent used significantly higher stimulation doses [14, 16, 17, 21], and also with the stimulation results from conventional infertility treatment. The fertility rate in patients who, together with their partner, decided upon cryoconservation of the fertilised egg cell was $61.3 \%$ per removed oocyte in the total collective. 
To compare efficacy, data from the German IVF Register (DIR) were consulted. The DIR collects data from all German centres for reproductive medicine. 40,816 IVF/ ICSI treatment cycles were performed in 2008; a mean of 9.5 oocytes were collected per stimulation cycles independent of age, and the fertilisation rate was $58.6 \%$ after IVF and $66.7 \%$ after ICSI [22].

Analysis of counselling and treatment data from the FertiPROTEKT network shows that primarily women who are childless at the time of their treatment access counselling and treatment with fertility preservation techniques. The techniques can be performed, alone or in combination, with a low risk of acute complications occurring. For ovarian stimulation in particular, the mean success rate and also the high range can be quantified using this data. Finally, the data emphasise that fertility preservation treatment can be and should be individualised and ideally performed as a combination of different techniques in order to increase its efficacy.

Acknowledgments We would like to thank all members of the FertiPROTEKT network for data acquisition. A list of all members is available on the network's website, under http://www.fertiprotekt.eu. We are greatly indebted to Mrs. Elizabeth Kraemer for reviewing our manuscript.

Conflict of interest We declare that we have no conflict of interest.

\section{References}

1. Schover LR, Rybicke LA, Martin BA, Bringelsen KA (1999) Having children after cancer. A pilot survey of survivors' attitude and experiences. Cancer 86:697-709

2. Schover LR, Brey K, Lichtin A, Lipshultz LI, Jeha S (2002) Knowledge and experience regarding cancer, infertility, and sperm banking in younger male survivors. J Clin Oncol 20: 1880-1889

3. Quinn GP, Vadaparampil ST, Ji-Hyun L, Jacobsen PB, Bepler G, Lancaster J, Keete DL, Albrecht TL (2009) Physician referral for fertility preservation in oncology patients: a national study of practice behaviors. J Clin Oncol 27:5952-5957

4. Beck-Fruchter R, Weiss A, Shalec E (2008) GnRH agonist therapy as ovarian protectants in female patients undergoing chemotherapy: a review of the clinical data. Hum Reprod Update 14(6):553-561

5. Blumenfeld Z, von Wolff M (2008) GnRH-analogues and oral contraceptives for fertility preservation in women during chemotherapy. Hum Reprod Update 14(6):543-552

6. Oktay K, Karlikaya G (2000) Ovarian function after transplantation of frozen, banked autologous ovarian tissue. N Engl J Med 342:1919

7. Lee SJ, Schover L, Partridge AH, Patrizio P, Wallace W, Hagerty R, Beck LN, Brennan LV, Oktay K (2006) American Society of Clinical Oncology recommendations on fertility preservation in cancer patients. J Clin Oncol 24:2917-2931
8. Huober-Zeeb C, Lawrenz B, Popovici R, Strowitzki T, Germeyer A, Stute P, v Wolff M (2010) Improving fertility preservation in cancer: ovarian tissue cryobanking followed by ovarian stimulation can be efficiently combined. Fertil Steril (in press)

9. FertiPROTEKT (2009) Network for fertility preservation before chemo- and radiotherapy. Available at: http://www.fertiprotekt.de/ index.php?lang=uk. Accessed 13 November 2009

10. Von Wolff M, Thaler C, Frambach T, Zeeb C, Lawrenz B, Popovici R, Strowitzki T (2009) Ovarian stimulation to crypopreserve fertilized oocytes in cancer patients can be started in the luteal phase. Fertil Steril 92(4):1360-1365

11. Behringer K, Breuer K, Reineke T, May M, Nogova L, Klimm B, Schmitz T, Wildt L, Diehl V, Engert A, German Hodgkin's Lymphoma Study Group (2005) Secondary amenorrhea after Hodgkin's lymphoma is influenced by age at treatment, stage of disease, chemotherapy regimen, and the use of oral contraceptives during therapy: a report from the German Hodgkin's Lymphoma Study Group. J Clin Oncol 23:7555-7564

12. Walshe JM, Denduluri N, Swain S (2006) Amenorrhea in premenopausal women after adjuvant chemotherapy for breast cancer. J Clin Oncol 24:5769-5779

13. Bines J, Oleske DM, Cobleigh MA (1996) Ovarian function in premenopausal women treated with adjuvant chemotherapy for breast cancer. J Clin Oncol 14:1718-1729

14. Klock SC, Zhang JX, Kazer R (2010) Fertility preservation for female cancer patients: early clinical experience. Fertil Steril 94(1):149-155

15. Rosendahl M, Andersen CY, Ernst E, Westergaard LG, Rasmussen PE, Loft A, Andersen AN (2008) Ovarian function after removal of an entire ovary for cryopreservation of pieces of cortex prior to gonadotoxic treatment: a follow-up study. Hum Reprod 23(11):2475-2483

16. Quintero RB, Helmer A, Huang JQ, Westphal LM (2010) Ovarian stimulation for fertility preservation in patients with cancer. Fertil Steril 93:865-868

17. Quintero RB, Helmer A, Murray E, Westphal LM (2005) Fertility preservation in female cancer patients. Fertil Steril 84(1):S70S71

18. Oktay K, Sahin G, Ucar KF, Barad D, Blanchette H, Gleicher N (2008) Fertility preservation is an emerging discipline extending beyond cancer patients: Analysis of a large experience. Fertil Steril 90(1):S51

19. Elassar A, Oktem O, Oktay K (2007) Fertility preservation with ovarian cryopreservation: a decade of experience. Fertil Steril 88(1):S12-S13

20. Oktay K, Oktem O (2010) Ovarian cryopreservation and transplantation for fertility preservation for medical indications: report of an ongoing experience. Fertil Steril 93:762-768

21. Knopmann J, Noyes N, Talebian S, Krey LC, Grifo JA, Licciardi F (2009) Women with cancer undergoing ART for fertility preservation: a cohort study of their response to exogenous gonadotropins. Fertil Steril 91(4):1476-1478

22. http://www.deutsches-ivf-register.de. Jahrbuch Deutsches IVFRegister 2008

23. Dittrich R, Maltaris T, Hoffmann I, Oppelt PG, Beckmann W, Mueller A (2010) Fertility preservation in cancer patients. Minerva Ginecol 62(1):63-80

24. Von Wolff M, Donnez J, Hovatta O, Keros V, Maltaris T, Montag M, Salle B, Sonmezer M, Andersen CY (2009) Cryopreservation and autotransplantation of human ovarian tissue prior to cytotoxic therapy-A technique in its infancy but already successful in fertility preservation. Eur J Cancer 45(9):1547-1553 